\title{
Trace Element Levels in Prostate Gland as Carcinoma's Markers
}

\author{
Vladimir Zaichick ${ }^{{ }^{*}}$, Sofia Zaichick ${ }^{2}$ \\ ${ }^{1}$ Radionuclide Diagnostics Department, Medical Radiological Research Centre, Obninsk, Russia \\ ${ }^{2}$ Laboratory of Dr. G.C. Piso, Northwestern University, Feinberg School of Medicine, Chicago, IL, USA \\ Email: *vezai@obninsk.com
}

How to cite this paper: Zaichick, V. and Zaichick, S. (2017) Trace Element Levels in Prostate Gland as Carcinoma's Markers. Journal of Cancer Therapy, 8, 131-145. https://doi.org/10.4236/jct.2017.82011

Received: January 9, 2017

Accepted: February 7, 2017

Published: February 10, 2017

Copyright $\odot 2017$ by authors and Scientific Research Publishing Inc. This work is licensed under the Creative Commons Attribution International License (CC BY 4.0).

http://creativecommons.org/licenses/by/4.0/

\begin{abstract}
Objectives: The aim of this study was to evaluate the changes in the prostatic levels of trace elements in the malignant human prostate. Methods: Contents of 43 trace elements in normal $(\mathrm{N}, \mathrm{n}=37)$, benign hypertrophic $(\mathrm{BPH}, \mathrm{n}=$ 32) and cancerous ( $\mathrm{PCa}, \mathrm{n}=60)$ prostate were investigated. Measurements were performed using instrumental neutron activation analysis and inductively coupled plasma mass spectrometry. Results: The mass fractions of all trace elements with the exception of $\mathrm{La}, \mathrm{Nb}$, and $\mathrm{Yb}$ show significant variations in cancerous prostate when compared with normal and BPH prostate. The contents of $\mathrm{Co}, \mathrm{Hg}, \mathrm{Rb}, \mathrm{Sc}, \mathrm{Se}$, and $\mathrm{Zn}$ were significantly lower and those of $\mathrm{Ag}, \mathrm{Al}, \mathrm{Au}, \mathrm{B}, \mathrm{Be}, \mathrm{Bi}, \mathrm{Br}, \mathrm{Ce}, \mathrm{Cr}, \mathrm{Cs}, \mathrm{Dy}, \mathrm{Er}, \mathrm{Gd}, \mathrm{Ho}, \mathrm{Li}, \mathrm{Mn}, \mathrm{Mo}, \mathrm{Nd}, \mathrm{Ni}$, $\mathrm{Pb}, \mathrm{Pr}, \mathrm{Sb}, \mathrm{Sm}, \mathrm{Sn}, \mathrm{Tb}, \mathrm{Th}, \mathrm{Ti}, \mathrm{Tl}, \mathrm{Tm}, \mathrm{U}, \mathrm{Y}, \mathrm{Yb}$, and $\mathrm{Zr}$ were significantly higher in PCa than in $\mathrm{BPH}$ tissues. When trace elements of cancerous prostate were compared with those in normal prostatic parenchyma, contents of $\mathrm{Cd}$, $\mathrm{Rb}, \mathrm{Sc}, \mathrm{Se}$, and $\mathrm{Zn}$ were significantly lower and $\mathrm{Ag}, \mathrm{Al}, \mathrm{Au}, \mathrm{B}, \mathrm{Be}, \mathrm{Bi}, \mathrm{Br}, \mathrm{Ce}$, Cr, Dy, Er, Fe, Gd, Hg, Ho, Li, Mn, Nd, Ni, Pr, Sb, Sm, Sn, Tb, Th, Tl, Y, and $\mathrm{Zr}$ were significantly higher. Conclusion: The $\mathrm{Ag}, \mathrm{Al}, \mathrm{B}, \mathrm{Br}, \mathrm{Li}, \mathrm{Mn}, \mathrm{Ni}$, and $\mathrm{Zn}$ mass fraction in a needle-biopsy core can be used as the informative indicators for distinguishing malignant from benign prostate. Sensitivity, specificity, and accuracy of these tests were in range $72 \%-100 \%, 66 \%-100 \%$, and $74 \%-98 \%$, respectively.
\end{abstract}

\section{Keywords}

Trace Elements, Prostate, Benign Prostatic Hypertrophy, Prostatic Carcinoma, Neutron Activation Analysis, Inductively Coupled Plasma Mass Spectrometry

\section{Introduction}

The prostate gland may be a source of many health problems in men past middle 
age, such as the most common benign prostatic hyperplasia (BPH), and prostatic carcinoma ( $\mathrm{PCa}) . \mathrm{BPH}$ is a noncancerous enlargement of the prostate gland leading to obstruction of the urethra and can significantly impair quality of life. The prevalence of histological BPH is found in approximately $50 \%-60 \%$ of males age $40-50$, in over $70 \%$ at 60 years old and in greater than $90 \%$ of men over 70 [1] [2]. In many Western industrialized countries, including North America, PCa is the most frequently diagnosed form of noncutaneous malignancy in males. Except for lung cancer, PCa is the leading cause of death from cancer [3]-[8]. Although the etiology of BPH and PCa is unknown, some trace elements have been highlighted in the literature in relation to the development of these prostate diseases [9]-[29].

Trace elements have essential physiological functions such as maintenance and regulation of cell function and signalling, gene regulation, activation or inhibition of enzymatic reactions, neurotransmission, and regulation of membrane function. Essential or toxic (mutagenic, carcinogenic) properties of trace elements depend on tissue-specific need or tolerance, respectively [30]. Excessive accumulation, deficiency or an imbalance of the trace elements may disturb the cell functions and may result in cellular degeneration, death and malignant transformation [30].

In earlier reported studies [31]-[64] significant changes of some trace element contents in hyperplastic and cancerous prostate in comparison with those in the normal prostatic tissue were observed. Moreover, a significant informative value of $\mathrm{Zn}$ content as a tumor marker for PCa diagnostics was shown by us [65] [66]. Hence some other trace elements besides $\mathrm{Zn}$ probably can be used as tumor markers for distinguishing between benign and malignant prostate.

Currently a number of methods were applied for the measurement of chemical elements contents in samples of human tissue. Among these methods, the instrumental neutron activation analysis with high resolution spectrometry of long-lived radionuclides (INAA-LLR) is non-destructive and one of the most sensitive techniques. It allows to measure the trace element contents in few milligrams tissue without any treatment of sample. Analytical studies of the Ag, Co, $\mathrm{Cr}, \mathrm{Fe}, \mathrm{Hg}, \mathrm{Sb}, \mathrm{Sc}, \mathrm{Se}$, and $\mathrm{Zn}$ contents in normal, $\mathrm{BPH}$ and $\mathrm{PCa}$ tissue were done by us using INAA-LLR [15] [20] [28] [60] [62]. Nondestructive method of analysis avoids the possibility of changing the content of trace elements in the studied samples [67] [68] [69] [70], which allows for the first time to obtain reliable results. In particular, it was shown that the average mass fraction of $\mathrm{Co}, \mathrm{Cr}, \mathrm{Hg}$, $\mathrm{Sb}$, and $\mathrm{Se}$ in $\mathrm{BPH}$ was higher than normal levels [66]. In PCa tissues the mean values of $\mathrm{Ag}, \mathrm{Cr}, \mathrm{Fe}, \mathrm{Hg}$, and $\mathrm{Sb}$ were higher while those of $\mathrm{Co}, \mathrm{Rb}, \mathrm{Sc}$, and $\mathrm{Zn}$ were lower than in healthy prostatic tissue [60] [66]. Obtained results formed the basis for a new method for differential diagnosis of BPH and PCa, the essence of which was to determine the content of trace elements in the material of needle biopsy of prostate indurated site.

It is obvious that the most effective methods will be non-destructive analytical methods because they involve a minimal treatment of sample since the chances 
of significant loss or contamination would be decreased. However, the INAALLR allow only to determine the mean mass fractions of 9 - 10 trace elements in the samples of normal and cancerous prostate glands [15] [20] [28] [60] [66]. The inductively coupled plasma mass spectrometry (ICP-MS) is a more power analytical tool than INAA-LLR [18] but sample digestion is a critical step in elemental analysis by this method. In the present study both analytical methods were used and the results obtained for some trace elements by ICP-MS were under the control of INAA-LLR data.

The present study had three aims. The main objective was to obtain reliable results about the 43 trace elements: $\mathrm{Ag}, \mathrm{Al}, \mathrm{Au}, \mathrm{B}, \mathrm{Be}, \mathrm{Bi}, \mathrm{Br}, \mathrm{Cd}, \mathrm{Ce}, \mathrm{Co}, \mathrm{Cr}, \mathrm{Cs}$, Dy, Er, Fe, Gd, Hg, Ho, La, Li, Mn, Mo, Nb, Nd, Ni, Pb, Pr, Rb, Sb, Sc, Se, Sm, $\mathrm{Sn}, \mathrm{Tb}, \mathrm{Th}, \mathrm{Ti}, \mathrm{Tl}, \mathrm{Tm}, \mathrm{U}, \mathrm{Y}, \mathrm{Yb}, \mathrm{Zn}$, and $\mathrm{Zr}$ contents in intact prostate of healthy men and in the prostate gland of BPH and PCa patients combining in consecutive order non-destructive INAA-LLR with destructive ICP-MS. The second aim was to compare the levels of trace elements in normal, hyperplastic, and cancerous prostate, and the third aim was to evaluate the trace element contents for diagnosis of prostate cancer.

All studies were approved by the Ethical Committee of the Medical Radiological Research Center, Obninsk.

\section{Materials and Methods}

\subsection{Materials}

The patients studied $(\mathrm{n}=92)$ were hospitalized in the Urological Department of the Medical Radiological Research Centre (Obninsk, Russia). All of them were European-Caucasian, citizens of Moscow and Obninsk (a small city in a non-industrial region $105 \mathrm{~km}$ south-west of Moscow). Transrectal puncture biopsy of suspicious indurated regions of the prostate was performed for every patient, to permit morphological study of prostatic tissue at these sites and to estimate their chemical element contents. For all patients the diagnosis has been confirmed by clinical and morphological results obtained during studies of biopsy and resected materials. The age of 32 patients with BPH ranged from 56 to 78 years, the mean being $66 \pm 6(\mathrm{M} \pm \mathrm{SD})$ years. The 60 patients aged $40-79$ suffered from PCa (stage T1 - T4). Their mean age was $65 \pm 10(\mathrm{M} \pm \mathrm{SD})$ years. There were no any inclusion and exclusion criteria of patients. Samples of prostate tissue were obtained from all patients with BPH and PCa, who were in the hospital of Medical Radiological Research Center in period of this study.

Intact prostates $(\mathrm{N})$ were removed at necropsy from 37 men aged $41-87$ who had died suddenly. All deceased were European-Caucasian, citizens of Moscow. Their mean age was $55 \pm 11(\mathrm{M} \pm \mathrm{SD})$ years. The majority of deaths were due to trauma. Tissue samples were collected from the peripheral zone of dorsal and lateral lobes of their prostates, within 2 days of death and then the samples were divided into two portions. One was used for morphological study while the other was intended for trace element analysis. A histological examination was used to control the age norm conformity, as well as to confirm the absence of micro- 
adenomatosis and latent cancer [15] [20] [28].

All tissue samples were divided into two portions. One was used for morphological study while the other was intended for trace element analysis. After the samples intended for trace element analysis were weighed, they were freeze-dried and homogenized. The sample weighing about $10 \mathrm{mg}$ (for biopsy materials) and 50 - $100 \mathrm{mg}$ (for resected materials) was used for trace element measurement by INAA-LLR. The samples for INAA-LLR were wrapped separately in a high-purity aluminum foil washed with double rectified alcohol beforehand and placed in a nitric acid-washed quartz ampoule.

After NAA-LLR investigation the prostate samples were taken out and used for ICP-MS. The samples were decomposed in autoclaves; $1.5 \mathrm{~mL}$ of concentrated $\mathrm{HNO}_{3}$ (nitric acid at $65 \%$, maximum (max) of $0.0000005 \% \mathrm{Hg}$; GR, ISO, Merck) and $0.3 \mathrm{~mL}$ of $\mathrm{H}_{2} \mathrm{O}_{2}$ (pure for analysis) were added to prostate tissue samples, placed in one-chamber autoclaves (Ancon-AT2, Ltd., Russia) and then heated for $3 \mathrm{~h}$ at $160^{\circ} \mathrm{C}-200^{\circ} \mathrm{C}$. After autoclaving, they were cooled to room temperature and solutions from the decomposed samples were diluted with deionized water (up to $20 \mathrm{~mL}$ ) and transferred to plastic measuring bottles. Simultaneously, the same procedure was performed in autoclaves without tissue samples (only $\mathrm{HNO}_{3}+\mathrm{H}_{2} \mathrm{O}_{2}+$ deionized water), and the resultant solutions were used as control samples.

\subsection{Methods}

A vertical channel of a nuclear reactor was applied to determine the trace element mass fractions by NAA-LLR. The quartz ampoule with prostate samples and certified reference materials was soldered, positioned in a transport aluminum container and exposed to a 24-hour neutron irradiation in a vertical channel with a neutron flux of $1 \cdot 3 \cdot 10^{13} \mathrm{n} \cdot \mathrm{cm}^{-2} \cdot \mathrm{s}^{-1}$. Ten days after irradiation samples were reweighed and repacked. The samples were measured for period from 10 to 30 days after irradiation. The duration of measurements was from $20 \mathrm{~min}$ to 10 hours subject to pulse counting rate. The gamma spectrometer included the 100 $\mathrm{cm}^{3} \mathrm{Ge}(\mathrm{Li})$ detector and on-line computer-based multichannel analyzer. The spectrometer provided a resolution of $1.9 \mathrm{keV}$ on the ${ }^{60} \mathrm{Co} 1332 \mathrm{keV}$ line.

An ICP-MS Thermo-Fisher "X-7" Spectrometer (Thermo Electron, USA) was used to determine the content of trace elements by ICP-MS. The element concentrations in aqueous solutions were determined by the quantitative method using multi elemental calibration solutions ICP-MS-68A and ICP-AM-6-A produced by High-Purity Standards (Charleston, SC 29423, USA). Indium was used as an internal standard in all measurements.

Information detailing with the NAA-LLR and ICP-MS methods used and other details of the analysis was presented in our previous publication [15] [20] [28] [60] [66].

For quality control, ten subsamples of the certified reference materials IAEA H-4 Animal muscle from the International Atomic Energy Agency (IAEA), as well as five sub-samples INCT-SBF-4 Soya Bean Flour, INCT-TL-1 Tea Leaves 
and INCT-MPH-2 Mixed Polish Herbs from the Institute of Nuclear Chemistry and Technology (INCT, Warszawa, Poland) were analyzed simultaneously with the investigated prostate tissue samples. All samples of CRM were treated in the same way as the prostate samples. Detailed results of this quality assurance program were presented in earlier publications [15] [18] [22].

A dedicated computer program for INAA mode optimization was used [71]. All prostate samples for INAA-LLR were prepared in duplicate and mean values of chemical element contents were used in final calculation. For elements investigated by INAA-LLR and ICP-MS the mean of all results was used. Using the Microsoft Office Excel software arithmetic mean, standard deviation, and standard error of mean was calculated for trace element mass fraction in normal, benign hyperplastic and cancerous prostate tissue. The difference in the results between BPH and Norm, PCa and Norm, as well as PCA and BPH was evaluated by parametric Student's $t$-test and non-parametric Wilcoxon-Mann-Whitney $U$-test. Values of $p<0.05$ were considered to be statistically significant. For the construction of "individual data sets for trace element mass fraction in normal, benign hypertrophic and cancerous prostate" diagrams the Microsoft Office Excel software was also used.

\section{Results}

Table 1 depicts mean values \pm standard error of mean $(\mathrm{M} \pm \mathrm{SEM})$ of the $\mathrm{Ag}, \mathrm{Al}$, $\mathrm{Au}, \mathrm{B}, \mathrm{Be}, \mathrm{Bi}, \mathrm{Br}, \mathrm{Cd}, \mathrm{Ce}, \mathrm{Co}, \mathrm{Cr}, \mathrm{Cs}, \mathrm{Dy}, \mathrm{Er}, \mathrm{Fe}, \mathrm{Gd}, \mathrm{Hg}, \mathrm{Ho}, \mathrm{La}, \mathrm{Li}, \mathrm{Mn}, \mathrm{Mo}, \mathrm{Nb}$, $\mathrm{Nd}, \mathrm{Ni}, \mathrm{Pb}, \mathrm{Pr}, \mathrm{Rb}, \mathrm{Sb}, \mathrm{Sc}, \mathrm{Se}, \mathrm{Sm}, \mathrm{Sn}, \mathrm{Tb}, \mathrm{Th}, \mathrm{Ti}, \mathrm{Tl}, \mathrm{Tm}, \mathrm{U}, \mathrm{Y}, \mathrm{Yb}, \mathrm{Zn}$ and Zr mass fraction in normal, benign hypertrophic and cancerous prostate.

The ratios of means and the difference between mean values of $\mathrm{Ag}, \mathrm{Al}, \mathrm{Au}, \mathrm{B}$, $\mathrm{Be}, \mathrm{Bi}, \mathrm{Br}, \mathrm{Cd}, \mathrm{Ce}, \mathrm{Co}, \mathrm{Cr}, \mathrm{Cs}, \mathrm{Dy}, \mathrm{Er}, \mathrm{Fe}, \mathrm{Gd}, \mathrm{Hg}, \mathrm{Ho}, \mathrm{La}, \mathrm{Li}, \mathrm{Mn}, \mathrm{Mo}, \mathrm{Nb}, \mathrm{Nd}$, $\mathrm{Ni}, \mathrm{Pb}, \mathrm{Pr}, \mathrm{Rb}, \mathrm{Sb}, \mathrm{Sc}, \mathrm{Se}, \mathrm{Sm}, \mathrm{Sn}, \mathrm{Tb}, \mathrm{Th}, \mathrm{Ti}, \mathrm{Tl}, \mathrm{Tm}, \mathrm{U}, \mathrm{Y}, \mathrm{Yb}, \mathrm{Zn}$ and $\mathrm{Zr}$ mass fraction in normal, benign hypertrophic and cancerous prostate are presented in Table 2.

Individual data sets for $\mathrm{Ag}, \mathrm{Al}, \mathrm{B}, \mathrm{Br}, \mathrm{Li}, \mathrm{Mn}, \mathrm{Ni}$ and $\mathrm{Zn}$ mass fraction in all investigated samples of normal, benign hypertrophic and cancerous prostate, respectively, are shown in Figure 1.

Table 3 contains parameters of the importance (sensitivity, specificity and accuracy) of $\mathrm{Ag}, \mathrm{Al}, \mathrm{B}, \mathrm{Br}, \mathrm{Li}, \mathrm{Mn}, \mathrm{Ni}$ and $\mathrm{Zn}$ mass fraction for the diagnosis of PCa calculated in this work.

\section{Discussion}

As was shown by us [15] [18] [22], the use of CRM IAEA H-4, INCT-SBF-4 Soya Bean Flour, INCT-TL-1 Tea Leaves, and INCT-MPH-2 Mixed Polish Herbs as certified reference materials for the analysis of samples of prostate tissue can be seen as quite acceptable. Good agreement of the $\mathrm{Ag}, \mathrm{Al}, \mathrm{Au}, \mathrm{B}, \mathrm{Be}, \mathrm{Bi}, \mathrm{Br}, \mathrm{Cd}, \mathrm{Ce}$, $\mathrm{Co}, \mathrm{Cr}, \mathrm{Cs}, \mathrm{Dy}, \mathrm{Er}, \mathrm{Fe}, \mathrm{Gd}, \mathrm{Hg}, \mathrm{Ho}, \mathrm{La}, \mathrm{Li}, \mathrm{Mn}, \mathrm{Mo}, \mathrm{Nb}, \mathrm{Nd}, \mathrm{Ni}, \mathrm{Pb}, \mathrm{Pr}, \mathrm{Rb}, \mathrm{Sb}$, Sc, Se, Sm, Sn, Tb, Th, Ti, Tl, Tm, U, Y, Yb, Zn and Zr contents analyzed by INAA-LLR and ICP-MS with the certified data of reference materials indicates 
Table 1. Comparison of mean values $(\mathrm{M} \pm \mathrm{SEM})$ of the trace element mass fraction $(\mathrm{mg} / \mathrm{kg}$, dry mass basis) in normal $(\mathrm{N})$, benign hypertrophic (BPH) and cancerous prostate (PCa).

\begin{tabular}{|c|c|c|c|c|}
\hline \multirow[b]{2}{*}{ Element } & \multirow[b]{2}{*}{ Symbol } & \multicolumn{3}{|c|}{ Prostatic tissue } \\
\hline & & $\begin{array}{c}\text { Normal } \\
41-87 \text { year }(n=37)\end{array}$ & $\begin{array}{c}\text { BPH } \\
56-78 \text { year }(\mathrm{n}=32)\end{array}$ & $\begin{array}{c}\text { PCa } \\
40-79 \text { year }(\mathrm{n}=60)\end{array}$ \\
\hline Silver & $\mathrm{Ag}$ & $0.038 \pm 0.006$ & $0.0415 \pm 0.0090$ & $0.252 \pm 0.030$ \\
\hline Aluminum & $\mathrm{Al}$ & $34.2 \pm 3.5$ & $24.4 \pm 3.2$ & $328 \pm 73$ \\
\hline Gold & $\mathrm{Au}$ & $0.0041 \pm 0.0008$ & $0.00257 \pm 0.00077$ & $0.0297 \pm 0.0056$ \\
\hline Boron & $\mathrm{B}$ & $1.04 \pm 0.18$ & $1.51 \pm 0.26$ & $12.6 \pm 3,7$ \\
\hline Berillium & $\mathrm{Be}$ & $0.00094 \pm 0.00007$ & $0.000918 \pm 0.000042$ & $0.0137 \pm 0.0022$ \\
\hline Bismuth & $\mathrm{Bi}$ & $0.029 \pm 0.011$ & $0.140 \pm 0.042$ & $1.75 \pm 0.27$ \\
\hline Bromine & $\mathrm{Br}$ & $27.9 \pm 2.9$ & $30.6 \pm 3.4$ & $99.9 \pm 8.9$ \\
\hline Cadmium & $\mathrm{Cd}$ & $1.12 \pm 0.13$ & $1.07 \pm 0.43$ & $0.425 \pm 0.099$ \\
\hline Cerium & $\mathrm{Ce}$ & $0.0309 \pm 0.0050$ & $0.0128 \pm 0.0019$ & $0.101 \pm 0.013$ \\
\hline Cobalt & Co & $0.0467 \pm 0.0064$ & $0.0617 \pm 0.0084$ & $0.0336 \pm 0.0040$ \\
\hline Cromium & $\mathrm{Cr}$ & $0.56 \pm 0.08$ & $1.00 \pm 0.10$ & $2.34 \pm 0.32$ \\
\hline Cesium & Cs & $0.0339 \pm 0.0033$ & $0.0235 \pm 0.0025$ & $0.0389 \pm 0.0039$ \\
\hline Dysprosium & Dy & $0.00293 \pm 0.00049$ & $0.00156 \pm 0.00024$ & $0.00771 \pm 0.00110$ \\
\hline Erbium & Er & $0.00148 \pm 0.00023$ & $0.00072 \pm 0.00013$ & $0.00297 \pm 0.00038$ \\
\hline Iron & $\mathrm{Fe}$ & $111 \pm 9$ & $133 \pm 11$ & $165 \pm 15$ \\
\hline Gadolinium & $\mathrm{Gd}$ & $0.00290 \pm 0.00041$ & $0.00153 \pm 0.00027$ & $0.00945 \pm 0.00173$ \\
\hline Mercury & $\mathrm{Hg}$ & $0.052 \pm 0.008$ & $0.259 \pm 0.029$ & $0.122 \pm 0.019$ \\
\hline Holmium & Ho & $0.00057 \pm 0.00008$ & $0.00032 \pm 0.00005$ & $0.00178 \pm 0.00022$ \\
\hline Lanthanum & $\mathrm{La}$ & $0.080 \pm 0.020$ & $0.0385 \pm 0.0073$ & $0.969 \pm 0.537$ \\
\hline Lithium & $\mathrm{Li}$ & $0.0419 \pm 0.0055$ & $0.0385 \pm 0.0073$ & $0.251 \pm 0.054$ \\
\hline Manganese & $\mathrm{Mn}$ & $1.34 \pm 0.08$ & $1.19 \pm 0.09$ & $6.99 \pm 1.35$ \\
\hline Molybdenum & Mo & $0.282 \pm 0.038$ & $0.167 \pm 0.009$ & $0.298 \pm 0.035$ \\
\hline Niobium & $\mathrm{Nb}$ & $0.0054 \pm 0.0012$ & $0.0102 \pm 0.0079$ & $0.0052 \pm 0.0002$ \\
\hline Neodymium & $\mathrm{Nd}$ & $0.0137 \pm 0.0021$ & $0.0062 \pm 0.0009$ & $0.0413 \pm 0.0065$ \\
\hline Nickel & $\mathrm{Ni}$ & $3.10 \pm 0.51$ & $3.22 \pm 1.06$ & $6.96 \pm 1.04$ \\
\hline Lead & $\mathrm{Pb}$ & $2.39 \pm 0.56$ & $0.69 \pm 0.16$ & $1.81 \pm 0.35$ \\
\hline Praseodymium & $\operatorname{Pr}$ & $0.00353 \pm 0.00053$ & $0.00149 \pm 0.00027$ & $0.00973 \pm 0.00174$ \\
\hline Rubidium & $\mathrm{Rb}$ & $13.3 \pm 0.9$ & $14.3 \pm 0.8$ & $8.71 \pm 0.66$ \\
\hline Antimony & $\mathrm{Sb}$ & $0.043 \pm 0.006$ & $0.163 \pm 0.036$ & $0.490 \pm 0.059$ \\
\hline Scandium & $\mathrm{Sc}$ & $0.0294 \pm 0.0053$ & $0.0257 \pm 0.0040$ & $0.0116 \pm 0.0015$ \\
\hline Selenium & $\mathrm{Se}$ & $0.75 \pm 0.05$ & $1.11 \pm 0.07$ & $0.56 \pm 0.08$ \\
\hline Samarium & $\mathrm{Sm}$ & $0.0027 \pm 0.0004$ & $0.0014 \pm 0.0004$ & $0.0095 \pm 0.0029$ \\
\hline Tin & Sn & $0.32 \pm 0.06$ & $0.108 \pm 0.029$ & $1.28 \pm 0.24$ \\
\hline Terbium & $\mathrm{Tb}$ & $0.00039 \pm 0.00006$ & $0.00017 \pm 0.00003$ & $0.00089 \pm 0.00012$ \\
\hline Thorium & Th & $0.0033 \pm 0.0007$ & $0.0018 \pm 0.0003$ & $0.0495 \pm 0.0123$ \\
\hline Titanium ${ }^{\star}$ & $\mathrm{Ti}^{*}$ & $2.82 \pm 0.64$ & $1.52 \pm 0.20$ & $8.60 \pm 2.20$ \\
\hline Thallium & $\mathrm{Tl}$ & $0.0014 \pm 0.0001$ & $0.00202 \pm 0.00057$ & $0.0219 \pm 0.0056$ \\
\hline Thulium & $\mathrm{Tm}$ & $0.00024 \pm 0.00003$ & $0.000151 \pm 0.000021$ & $0.000535 \pm 0.000111$ \\
\hline Uranium & $\mathrm{U}$ & $0.0070 \pm 0.0021$ & $0.0021 \pm 0.0009$ & $0.0068 \pm 0.0013$ \\
\hline Yttrium & $\mathrm{Y}$ & $0.0187 \pm 0.0043$ & $0.0071 \pm 0.0012$ & $0.0340 \pm 0.0038$ \\
\hline Ytterbium & $\mathrm{Yb}$ & $0.00141 \pm 0.00025$ & $0.00083 \pm 0.00020$ & $0.00174 \pm 0.00039$ \\
\hline Zinc & $\mathrm{Zn}$ & $1031 \pm 129$ & $1271 \pm 102$ & $136 \pm 10$ \\
\hline Zirconium & $\mathrm{Zr}$ & $0.036 \pm 0.006$ & $0.091 \pm 0.036$ & $2.13 \pm 0.89$ \\
\hline
\end{tabular}

$\mathrm{M}$-arithmetic mean, SEM—standard error of mean, ${ }^{\star}$ Titanium tools were used for sampling and sample preparation. 
Table 2. Ratio of means and the difference between mean values of trace element mass fractions in normal $(\mathrm{N})$, benign hypertrophic $(\mathrm{BPH})$ and cancerous prostate $(\mathrm{PCa})$.

\begin{tabular}{|c|c|c|c|c|c|c|c|c|c|}
\hline & \multicolumn{3}{|c|}{$\mathrm{BPH}$ and Normal $(\mathrm{N})$} & \multicolumn{3}{|c|}{ PCa and Normal (N) } & \multicolumn{3}{|c|}{$\mathrm{PCa}$ and $\mathrm{BPH}$} \\
\hline & $\begin{array}{c}\text { Ratio } \\
\text { BPH/N }\end{array}$ & $\begin{array}{c}p \leq \\
\text { t-test }\end{array}$ & $\begin{array}{c}p \\
\text { U-test }\end{array}$ & $\begin{array}{l}\text { Ratio } \\
\mathrm{PCa} / \mathrm{N}\end{array}$ & $\begin{array}{c}p \leq \\
\text { t-test }\end{array}$ & $\begin{array}{c}p \\
\text { U-test }\end{array}$ & $\begin{array}{c}\text { Ratio } \\
\text { PCa/BPH }\end{array}$ & $\begin{array}{c}p \leq \\
\text { t-test }\end{array}$ & $\begin{array}{c}p \\
\text { U-test }\end{array}$ \\
\hline $\mathrm{Ag}$ & 1.09 & 0.74 & $>0.05$ & 6.63 & 0.001 & $<0.01$ & 6.07 & 0.001 & $<0.01$ \\
\hline $\mathrm{Al}$ & 0.71 & 0.052 & $>0.05$ & 9.59 & 0.003 & $<0.01$ & 13.4 & 0.002 & $<0.01$ \\
\hline $\mathrm{Au}$ & 0.63 & 0.17 & $>0.05$ & 7.24 & 0.001 & $<0.01$ & 11.6 & 0.001 & $<0.01$ \\
\hline $\mathrm{B}$ & 1.45 & 0.16 & $>0.05$ & 12.1 & 0.012 & $<0.01$ & 8.34 & 0.015 & $<0.01$ \\
\hline $\mathrm{Be}$ & 0.98 & 0.78 & $>0.05$ & 14.6 & 0.001 & $<0.01$ & 14.9 & 0.001 & $<0.01$ \\
\hline $\mathrm{Bi}$ & 4.83 & 0.026 & $>0.05$ & 60.3 & 0.001 & $<0.01$ & 12.5 & 0.001 & $<0.01$ \\
\hline $\mathrm{Br}$ & 1.10 & 0.53 & $>0.05$ & 3.58 & 0.001 & $<0.01$ & 3.26 & 0.001 & $<0.01$ \\
\hline $\mathrm{Cd}$ & 0.96 & 0.95 & $>0.05$ & 0.38 & 0.001 & $<0.05$ & 0.40 & 0.17 & $>0.05$ \\
\hline $\mathrm{Ce}$ & 0.41 & 0.002 & $>0.05$ & 3,27 & 0.001 & $<0.01$ & 7.89 & 0.001 & $<0.01$ \\
\hline Co & 1.32 & 0.16 & $>0.05$ & 0.72 & 0.087 & $>0.05$ & 0.54 & 0.005 & $>0.05$ \\
\hline $\mathrm{Cr}$ & 1.79 & 0.002 & $<0.01$ & 4.18 & 0.001 & $<0.01$ & 2.34 & 0.001 & $<0.01$ \\
\hline Cs & 0.69 & 0.017 & $>0.05$ & 1.15 & 0.33 & $>0.05$ & 1.66 & 0.004 & $<0.01$ \\
\hline Dy & 0.53 & 0.018 & $>0.05$ & 2.63 & 0.003 & $<0.05$ & 4.94 & 0.001 & $<0.01$ \\
\hline $\mathrm{Er}$ & 0.49 & 0.007 & $>0.05$ & 2.01 & 0.004 & $<0.05$ & 4.13 & 0.001 & $<0.01$ \\
\hline $\mathrm{Fe}$ & 1.20 & 0.13 & $>0.05$ & 1.49 & 0.004 & $<0.05$ & 1.24 & 0.10 & $>0.05$ \\
\hline $\mathrm{Gd}$ & 0.53 & 0.008 & $>0.05$ & 3.26 & 0.004 & $<0.01$ & 6.18 & 0.001 & $<0.01$ \\
\hline $\mathrm{Hg}$ & 4.98 & 0.001 & $<0.01$ & 2.35 & 0.002 & $<0.01$ & 0.47 & 0.004 & $<0.01$ \\
\hline Ho & 0.56 & 0.012 & $>0.05$ & 3.12 & 0.001 & $<0.01$ & 5.56 & 0.001 & $<0.01$ \\
\hline $\mathrm{La}$ & 0.48 & 0.005 & $>0.05$ & 12.1 & 0.132 & $>0.05$ & 25.2 & 0.11 & $<0.01$ \\
\hline $\mathrm{Li}$ & 0.92 & 0.71 & $>0.05$ & 5.99 & 0.003 & $<0.01$ & 6.52 & 0.003 & $<0.01$ \\
\hline $\mathrm{Mn}$ & 0.89 & 0.25 & $>0.05$ & 5.22 & 0.002 & $<0.01$ & 5.87 & 0.002 & $<0.01$ \\
\hline Mo & 0.59 & 0.007 & $<0.05$ & 1.06 & 0.76 & $>0.05$ & 1.78 & 0.018 & $=0.05$ \\
\hline $\mathrm{Nb}$ & 1.89 & 0.56 & $>0.05$ & 0.96 & 0.82 & $>0.05$ & 0.51 & 0.53 & $>0.05$ \\
\hline $\mathrm{Nd}$ & 0.45 & 0.003 & $<0.01$ & 3.01 & 0.002 & $<0.01$ & 6.66 & 0.001 & $<0.01$ \\
\hline $\mathrm{Ni}$ & 1.04 & 0.92 & $>0.05$ & 2.25 & 0.005 & $<0.01$ & 2.16 & 0.020 & $<0.01$ \\
\hline $\mathrm{Pb}$ & 0.29 & 0.007 & $>0.05$ & 0.76 & 0.38 & $>0.05$ & 2.62 & 0.011 & $<0.01$ \\
\hline $\operatorname{Pr}$ & 0.42 & 0.002 & $<0.05$ & 2.76 & 0.005 & $<0.01$ & 6.53 & 0.001 & $<0.01$ \\
\hline $\mathrm{Rb}$ & 1.08 & 0.42 & $>0.05$ & 0.65 & 0.001 & $<0.01$ & 0.61 & 0.001 & $<0.01$ \\
\hline $\mathrm{Sb}$ & 3.79 & 0.004 & $>0.05$ & 11.4 & 0.001 & $<0.01$ & 3.01 & 0.001 & $<0.01$ \\
\hline $\mathrm{Sc}$ & 0.87 & 0.58 & $>0.05$ & 0.39 & 0.004 & $<0.01$ & 0.45 & 0.004 & $<0.01$ \\
\hline $\mathrm{Se}$ & 1.48 & 0.001 & $<0.05$ & 0.75 & 0.041 & $<0.05$ & 0.50 & 0.001 & $<0.01$ \\
\hline $\mathrm{Sm}$ & 0.52 & 0.023 & $>0.05$ & 3.52 & 0.039 & $<0.05$ & 6.79 & 0.019 & $<0.05$ \\
\hline Sn & 0.34 & 0.005 & $<0.01$ & 4.00 & 0.002 & $<0.01$ & 11.9 & 0.001 & $<0.01$ \\
\hline $\mathrm{Tb}$ & 0.44 & 0.002 & $<0.05$ & 2.28 & 0.008 & $<0.01$ & 5.24 & 0.003 & $<0.01$ \\
\hline Th & 0.55 & 0.066 & $>0.05$ & 15.0 & 0.004 & $<0.01$ & 27.5 & 0.003 & $<0.01$ \\
\hline $\mathrm{Ti}^{*}$ & 0.53 & 0.064 & $>0.05$ & 3.05 & 0.056 & $>0.05$ & 5.73 & 0.032 & $>0.05$ \\
\hline $\mathrm{Tl}$ & 1.44 & 0.30 & $>0.05$ & 15.6 & 0.004 & $<0.01$ & 10.8 & 0.005 & $<0.01$ \\
\hline $\mathrm{Tm}$ & 0.63 & 0.033 & $>0.05$ & 2.23 & 0.072 & $<0.05$ & 3.54 & 0.038 & $<0.01$ \\
\hline $\mathrm{U}$ & 0.30 & 0.041 & $>0.05$ & 0.97 & 0.95 & $>0.05$ & 3.24 & 0.008 & $<0.01$ \\
\hline $\mathrm{Y}$ & 0.38 & 0.014 & $>0.05$ & 1.82 & 0.012 & $<0.01$ & 4.79 & 0.001 & $<0.01$ \\
\hline $\mathrm{Yb}$ & 0.59 & 0.074 & $>0.05$ & 1.23 & 0.50 & $>0.05$ & 2.10 & 0.080 & $>0.05$ \\
\hline $\mathrm{Zn}$ & 1.23 & 0.15 & $>0.05$ & 0.13 & 0.001 & $<0.01$ & 0.11 & 0.001 & $<0.01$ \\
\hline $\mathrm{Zr}$ & 2.53 & 0.17 & $>0.05$ & 59.2 & 0.041 & $<0.05$ & 23.4 & 0.045 & $<0.01$ \\
\hline
\end{tabular}

$t$-test-Student's $t$-test, U-test-Wilcoxon-Mann-Whitney U-test, Bold significant differences. 


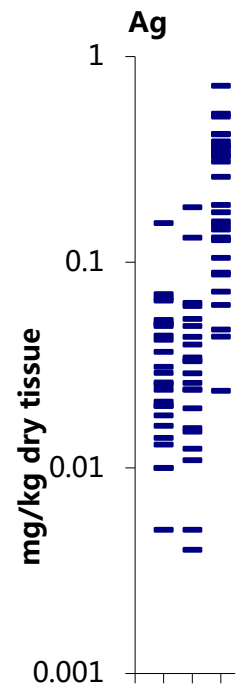

123

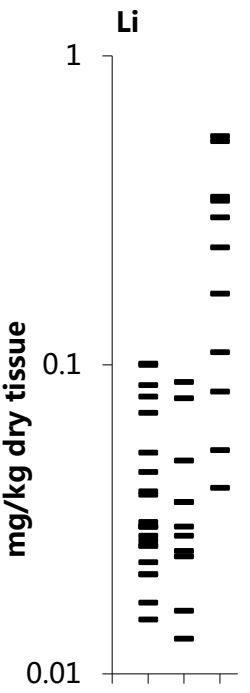

123

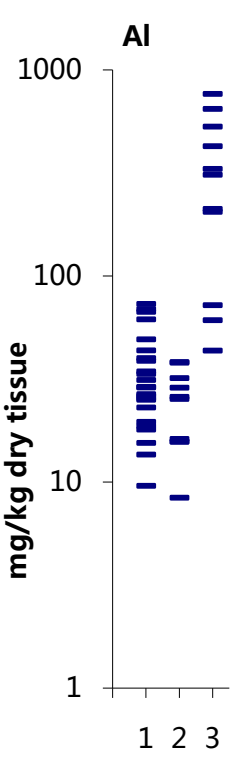

Mn

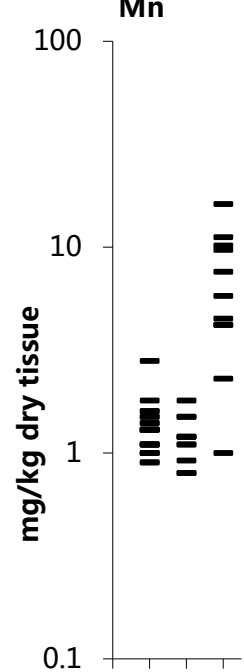

123

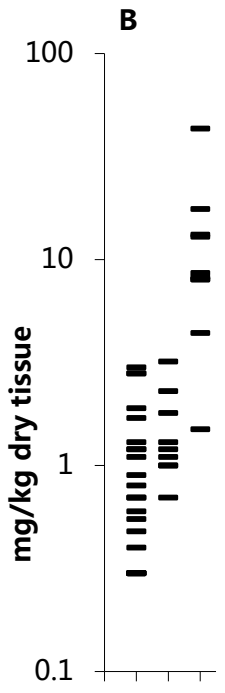

123

$\mathrm{Ni}$

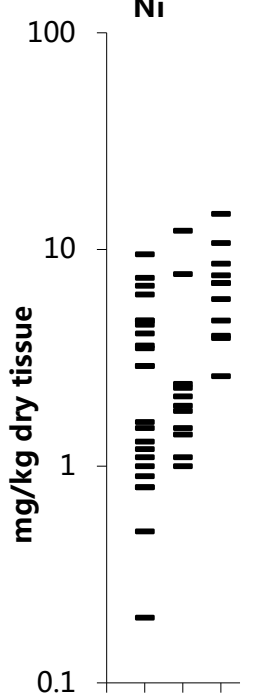

123

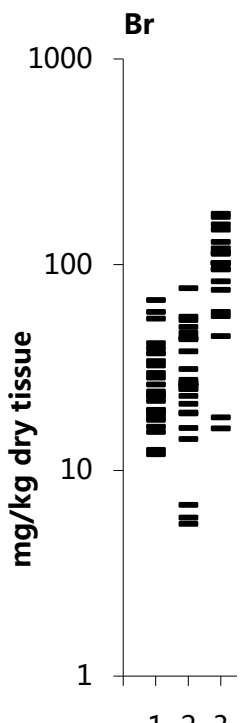

123

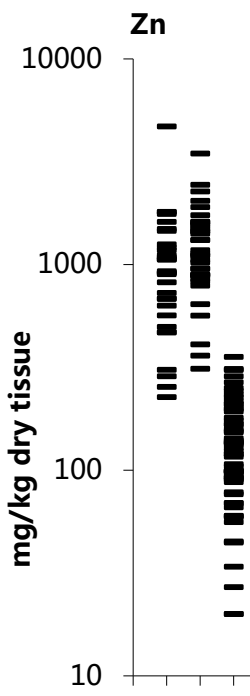

123

Figure 1. Individual data sets for $\mathrm{Ag}, \mathrm{Al}, \mathrm{B}, \mathrm{Br}, \mathrm{Li}, \mathrm{Mn}, \mathrm{Ni}$ and $\mathrm{Zn}$ mass fraction in samples of normal (1), benign hypertrophic (2) and cancerous (3) prostate.

Table 3. Parameters $(M \pm S D)$ of the importance (sensitivity, specificity and accuracy) of $\mathrm{Al}, \mathrm{B}, \mathrm{Mn}$, and $\mathrm{Zn}$ mass fraction for the diagnosis of $\mathrm{PCa}$ (estimation is made for "PCa or normal and BPH prostate").

\begin{tabular}{ccccc}
\hline Mass fraction & $\begin{array}{c}\text { Limit for PCa } \\
\mathrm{mg} / \mathrm{kg} \text { dry tissue }\end{array}$ & Sensitivity & Specificity & Accuracy \\
\hline $\mathrm{Ag}$ & $\geq 0.085$ & $88 \pm 6$ & $92 \pm 4$ & $92 \pm 3$ \\
$\mathrm{Al}$ & $\geq 100$ & $72 \pm 14$ & $100-3$ & $94 \pm 3$ \\
$\mathrm{~B}$ & $\geq 4.0$ & $90 \pm 10$ & $100-3$ & $98 \pm 2$ \\
$\mathrm{Br}$ & $\geq 60$ & $83 \pm 8$ & $92 \pm 4$ & $89 \pm 4$ \\
$\mathrm{Li}$ & $\geq 0.08$ & $82 \pm 12$ & $91 \pm 5$ & $89 \pm 4$ \\
$\mathrm{Mn}$ & $\geq 2.0$ & $91 \pm 9$ & $97 \pm 3$ & $96 \pm 3$ \\
$\mathrm{Ni}$ & $\geq 2.5$ & $100-9$ & $66 \pm 8$ & $74 \pm 7$ \\
$\mathrm{Zn}$ & $\leq 350$ & $99 \pm 1$ & $93 \pm 3$ & $96 \pm 2$ \\
\hline
\end{tabular}

$\mathrm{M}$-arithmetic mean, SD—standard deviation. 
an acceptable accuracy of the results obtained in the study of trace elements of the prostate samples presented in Table 1 and Table 2.

The mean values and all selected statistical parameters were calculated for 43 trace elements: $\mathrm{Ag}, \mathrm{Al}, \mathrm{Au}, \mathrm{B}, \mathrm{Be}, \mathrm{Bi}, \mathrm{Br}, \mathrm{Cd}, \mathrm{Ce}, \mathrm{Co}, \mathrm{Cr}, \mathrm{Cs}, \mathrm{Dy}, \mathrm{Er}, \mathrm{Fe}, \mathrm{Gd}, \mathrm{Hg}$, Ho, La, Li, Mn, Mo, Nb, Nd, Ni, Pb, Pr, Rb, Sb, Sc, Se, Sm, Sn, Tb, Th, Ti, Tl, $\mathrm{Tm}, \mathrm{U}, \mathrm{Y}, \mathrm{Yb}, \mathrm{Zn}$, and $\mathrm{Zr}$ trace element mass fractions (Table 1). The mass fraction of these trace elements were measured in all, or a major portion of normal prostate samples. The masses of $\mathrm{BPH}$ and $\mathrm{PCa}$ samples varied very strong from a few milligrams (sample from needle biopsy material) to $100 \mathrm{mg}$ (sample from resected material). Therefore, in $\mathrm{BPH}$ and $\mathrm{PCa}$ prostates mass fractions of $\mathrm{Ag}$, $\mathrm{Br}, \mathrm{Co}, \mathrm{Cr}, \mathrm{Fe}, \mathrm{Hg}, \mathrm{Rb}, \mathrm{Sb}, \mathrm{Sc}$, Se, and $\mathrm{Zn}$ were measured in all, or a major portion of samples, while mass fractions of other trace elements were determined in 22 samples (11 BPH and 11 PCa samples, respectively).

From Table 2, it is observed that in benign hypertrophic tissues the mass fractions of $\mathrm{Ag}, \mathrm{Al}, \mathrm{Au}, \mathrm{B}, \mathrm{Be}, \mathrm{Br}, \mathrm{Cd}, \mathrm{Co}, \mathrm{Fe}, \mathrm{Li}, \mathrm{Mn}, \mathrm{Nb}, \mathrm{Ni}, \mathrm{Rb}, \mathrm{Sc}, \mathrm{Th}, \mathrm{Ti}, \mathrm{Tl}, \mathrm{Yb}$, $\mathrm{Zn}$, and $\mathrm{Zr}$ not differ from normal levels, but the mass fractions of $\mathrm{Bi}, \mathrm{Cr}, \mathrm{Hg}, \mathrm{Sb}$, and Se are higher, while the mass fraction of Ce, Cs, Dy, Er, Gd, Ho, La, Mo, Nd, $\mathrm{Pb}, \mathrm{Pr}, \mathrm{Sm}, \mathrm{Sn}, \mathrm{Tb}, \mathrm{Tm}, \mathrm{U}$, and $\mathrm{Y}$ are significantly lower. In cancerous tissue the mass fractions of $\mathrm{Cd}, \mathrm{Rb}, \mathrm{Sc}, \mathrm{Se}$, and $\mathrm{Zn}$ are significantly lower, and mass fractions of $\mathrm{Ag}, \mathrm{Al}, \mathrm{Au}, \mathrm{B}, \mathrm{Be}, \mathrm{Bi}, \mathrm{Br}, \mathrm{Cd}, \mathrm{Ce}, \mathrm{Cr}, \mathrm{Dy}, \mathrm{Er}, \mathrm{Fe}, \mathrm{Gd}, \mathrm{Hg}, \mathrm{Ho}, \mathrm{Li}, \mathrm{Mn}, \mathrm{Nd}$, $\mathrm{Ni}, \mathrm{Pr}, \mathrm{Sb}, \mathrm{Sm}, \mathrm{Sn}, \mathrm{Tb}, \mathrm{Th}, \mathrm{Tl}, \mathrm{Y}$, and $\mathrm{Zr}$ are significantly higher than in normal tissues of the prostate. All these trace elements show similar variations in cancerous tissues when compared with benign hypertrophic tissues of the prostate.

Analysis of the trace element mass fraction in prostate tissue could become a powerful diagnostic tool. To a large extent, the resumption of the search for new methods for early diagnosis of PCa was due to experience gained in a critical assessment of the limited capacity of the prostate specific antigen (PSA) serum test [72]. In addition to the PSA serum test and morphological study of needle-biopsy cores of the prostate, the development of other highly precise testing methods seems to be very useful.

Experimental conditions of the present study were approximated to the hospital conditions as closely as possible. In BPH and PCa cases we analyzed a part of the material obtained from a puncture transrectal biopsy of the indurated site in the prostate. Therefore, our data allow us to evaluate adequately the importance of trace element mass fraction for the diagnosis of PCa. As is evident from Table 2 and, particularly, from individual data sets (Figure 1), the $\mathrm{Ag}, \mathrm{Al}, \mathrm{B}, \mathrm{Br}$, $\mathrm{Li}, \mathrm{Mn}, \mathrm{Ni}$ and $\mathrm{Zn}$ mass fraction are potentially the most informative test for a differential diagnosis. For example, if $4.0 \mathrm{mg} / \mathrm{kg}$ is the value of B mass fraction assumed to be the lower limit for PCa (Figure 1) and an estimation is made for "PCa or intact and BPH tissue", the following values are obtained:

Sensitivity $=\{$ True Positives (TP)/[TP + False Negatives $(\mathrm{FN})]\} \cdot 100 \%=90 \% \pm$ $10 \%$;

Specificity $=\{$ True Negatives $(\mathrm{TN}) /[\mathrm{TN}+$ False Positives $(\mathrm{FP})]\} \cdot 100 \%=100 \%$ $-3 \%$ 
Accuracy $=[(\mathrm{TP}+\mathrm{TN}) /(\mathrm{TP}+\mathrm{FP}+\mathrm{TN}+\mathrm{FN})] \cdot 100 \%=98 \% \pm 2 \%$.

The number of people (samples) examined was taken into account for calculation of confidence intervals [73]. In other words, if B mass fraction in a prostate biopsy sample is higher than $4 \mathrm{mg} / \mathrm{kg}$, one could diagnose a malignant tumor with an accuracy $98 \% \pm 2 \%$. Thus, using the $\mathrm{B}$ mass fraction-test makes it possible to diagnose cancer in $100 \%-3 \%$; cases (sensitivity). The same way parameters of the importance (sensitivity, specificity and accuracy) of $\mathrm{Ag}, \mathrm{Al}, \mathrm{B}, \mathrm{Br}, \mathrm{Li}$, $\mathrm{Mn}, \mathrm{Ni}$ and $\mathrm{Zn}$ mass fraction for the diagnosis of PCa were calculated (Table 3).

\section{Conclusions}

The combination of nondestructive INAALSLR and destructive ICP-MS methods is satisfactory analytical tool for the precise determination of 43 trace element mass fractions in the tissue samples of normal, $\mathrm{BPH}$ and carcinomatous prostate glands. The sequential application of two methods allowed precise quantitative determinations of mean mass fraction of $\mathrm{Ag}, \mathrm{Al}, \mathrm{Au}, \mathrm{B}, \mathrm{Be}, \mathrm{Bi}, \mathrm{Br}$, Cd, Ce, Co, Cr, Cs, Dy, Er, Fe, Gd, Hg, Ho, La, Li, Mn, Mo, Nb, Nd, Ni, Pb, Pr, $\mathrm{Rb}, \mathrm{Sb}, \mathrm{Sc}, \mathrm{Se}, \mathrm{Sm}, \mathrm{Sn}, \mathrm{Tb}, \mathrm{Th}, \mathrm{Ti}, \mathrm{Tl}, \mathrm{Tm}, \mathrm{U}, \mathrm{Y}, \mathrm{Yb}, \mathrm{Zn}$ and Zr. The mass fractions of all trace elements investigated in the study with the exception of $\mathrm{La}, \mathrm{Nb}$, and $\mathrm{Yb}$ show significant variations in cancerous tissues when compared with normal and $\mathrm{BPH}$ prostate. The contents of $\mathrm{Rb}, \mathrm{Sc}, \mathrm{Se}$, and $\mathrm{Zn}$ were significantly lower and those of $\mathrm{Ag}, \mathrm{Al}, \mathrm{Au}, \mathrm{B}, \mathrm{Be}, \mathrm{Bi}, \mathrm{Br}, \mathrm{Ce}, \mathrm{Cr}, \mathrm{Dy}, \mathrm{Er}, \mathrm{Fe}, \mathrm{Gd}, \mathrm{Ho}, \mathrm{Li}, \mathrm{Mn}$, $\mathrm{Nd}, \mathrm{Ni}, \mathrm{Pr}, \mathrm{Sb}, \mathrm{Sm}, \mathrm{Sn}, \mathrm{Tb}, \mathrm{Th}, \mathrm{Tl}, \mathrm{Y}$, and Zr were significantly higher in cancerous tissues than in normal and BPH tissues. The $\mathrm{Ag}, \mathrm{Al}, \mathrm{B}, \mathrm{Br}, \mathrm{Li}, \mathrm{Mn}, \mathrm{Ni}$ and $\mathrm{Zn}$ mass fraction in a needle-biopsy core can be used as an accurate tool to diagnose prostate cancer. Further studies on larger number of samples are required to confirm our findings, to study the impact of the trace element contents on prostate cancer etiology.

\section{Acknowledgements}

We are grateful to Dr. Tatyana Sviridova, Medical Radiological Research Center, Obninsk, and to the late Prof. A.A. Zhavoronkov, Institute of Human Morphology, Russian Academy of Medical Sciences, Moscow, for supplying prostate samples. We are also grateful to Dr. Karandaschev V., Dr. Nosenko S., and Moskvina I., Institute of Microelectronics Technology and High Purity Materials, Chernogolovka, Russia, for their help in ICP-MS analysis.

\section{References}

[1] Roehrborn, C. and McConnell, J. (2002) Etiology, Pathophysiology, Epidemiology and Natural History of Benign Prostatic Hyperplasia. In: Walsh, P., Retik, A., Vaughan, E. and Wein, A., Eds., Campbells Urology, 8th ed., Saunders, Philadelphia, 1297-1336.

[2] Lepor, H. (2005) Pathophysiology of Benign Prostatic Hyperplasia in the Aging Male Population. Reviews in Urology, 7, S3-S12.

[3] Oliver, S.E., Gunnell, D. and Donovan, J.L. (2000) Comparison of Trends in Prostate-Cancer Mortality in England and Wales and the USA. The Lancet, 355, 1788- 
1789. https://doi.org/10.1016/S0140-6736(00)02269-8

[4] Kumar, R.J., Barqawi, A.B. and Crawford, E.D. (2004) Epidemiology of Prostate Cancer. Business Briefing: US Oncology Review, 1-6.

[5] Maddams, J., Brewster, D., Gavin, A., Steward, J., Elliott, J., Utley, M. and Møller, H. (2009) Cancer Prevalence in the United Kingdom: Estimates for 2008. British Journal of Cancer, 101, 541-547. https://doi.org/10.1038/sj.bjc.6605148

[6] Lutz, J.M., Francisci, S., Mugno, E., Usel, M., Pompe-Kirn, V., Coebergh, J.W., Bieslka-Lasota, M. and EUROPREVAL Working Group (2003) Cancer Prevalence in Central Europe: The EUROPREVAL Study. Annals of Oncology, 14, 313-322. https://doi.org/10.1093/annonc/mdg059

[7] Möller, T., Anderson, H., Aareleid, T., Hakulinen, T., Storm, H., Tryggvadottir, L., Corazziari, I., Mugno, E. and EUROPREVAL Working Group (2003) Cancer Prevalence in Northern Europe: The EUROPREVAL Study. Annals of Oncology, 14, 946-957. https://doi.org/10.1093/annonc/mdg255

[8] De Angelis, R., Grande, E., Inghelmann, R., Francisci, S., Micheli, A., Baili, P., Meneghini, E., Capocaccia, R. and Verdecchia, A. (2007) Cancer Prevalence Estimates in Italy from 1970 to 2010. Tumori, 93, 392-397.

[9] Waalkes, M.P. and Rehm, S. (1994) Cadmium and Prostate Cancer. Journal of Toxicology and Environmental Health, 43, 251-269.

https://doi.org/10.1080/15287399409531920

[10] Zaichick, V. and Zaichick, S. (1999) Role of Zinc in Prostate Cancerogenesis. 19 Arbeitstagung on Mengen und Spurenelemente, Friedrich-Schiller-Universitat, Jena, 104-115.

[11] Platz, E.A. and Helzlsouer, K.J. (2001) Selenium, Zinc, and Prostate Cancer. Epidemiologic Reviews, 23, 93-101.

https://doi.org/10.1093/oxfordjournals.epirev.a000801

[12] Zaichick, V. (2004) INAA and EDXRF Applications in the Age Dynamics Assessment of $\mathrm{Zn}$ Content and Distribution in the normal Human Prostate. Journal of Radioanalytical and Nuclear Chemistry, 262, 229-234.

https://doi.org/10.1023/B:JRNC.0000040879.45030.4f

[13] Gray, M.A., Centeno, J.A., Slaney, D.P., Ejnik, J.W., Todorov, T. and Nacey, J.N. (2005) Environmental Exposure to Trace Elements and Prostate Cancer in Three New Zealand Ethnic Groups. International Journal of Environmental Research and Public Health, 2, 374-384. https://doi.org/10.3390/ijerph2005030001

[14] Zaichick, S. and Zaichick, V. (2011) INAA Application in the Age Dynamics Assessment of $\mathrm{Br}, \mathrm{Ca}, \mathrm{Cl}, \mathrm{K}, \mathrm{Mg}, \mathrm{Mn}$, and $\mathrm{Na}$ Content in the Normal Human Prostate. Journal of Radioanalytical and Nuclear Chemistry, 288, 197-202. https://doi.org/10.1007/s10967-010-0927-4

[15] Zaichick, S. and Zaichick, V. (2011) The Effect of Age on Ag, Co, Cr, Fe, Hg, Sb, Sc, $\mathrm{Se}$, and $\mathrm{Zn}$ Contents in Intact Human Prostate Investigated by Neutron Activation Analysis. Applied Radiation and Isotopes, 69, 827-833. https://doi.org/10.1016/j.apradiso.2011.02.010

[16] Zaichick, S. and Zaichick, V. (2011) The Br, Fe, Rb, Sr, and Zn Content and Interrelation in Intact and Morphologic Normal Prostate Tissue of Adult Men Investigated by Energy Dispersive X-Ray Fluorescent Analysis. X-Ray Spectrometry, 40, 464-469. https://doi.org/10.1002/xrs.1370

[17] Zaichick, V., Nosenko, S. and Moskvina, I. (2012) The Effect of Age on 12 Chemical Element Contents in Intact Prostate of Adult Men Investigated by Inductively Coupled Plasma Atomic Emission Spectrometry. Biological Trace Element Research, 147, 49-58. https://doi.org/10.1007/s12011-011-9294-4 
[18] Zaichick, S., Zaichick, V., Nosenko, S. and Moskvina, I. (2012) Mass fractions of 52 Trace Elements and Zinc Trace Element Content Ratios in Intact Human Prostates Investigated by Inductively Coupled Plasma Mass Spectrometry. Biological Trace Element Research, 149, 171-183. https://doi.org/10.1007/s12011-012-9427-4

[19] Zaichick, V. and Zaichick, S. (2014) Age-Related Histological and Zinc Content Changes in Adult Nonhyperplastic Prostate Glands. Age, 36, 167-181.

https://doi.org/10.1007/s11357-013-9561-8

[20] Zaichick, V. and Zaichick, S. (2014) INAA Application in the Assessment of Chemical Element Mass Fractions in Adult and Geriatric Prostate Glands. Applied Radiation and Isotopes, 90, 62-73. https://doi.org/10.1016/j.apradiso.2014.03.010

[21] Zaichick, V. and Zaichick, S. (2014) Determination of Trace Elements in Adults and Geriatric Prostate Combining Neutron Activation with Inductively Coupled Plasma Atomic Emission Spectrometry. Open Journal of Biochemistry, 1, 16-33.

[22] Zaichick, V. and Zaichick, S. (2014) Use of INAA and ICP-MS for the Assessment of Trace Element Mass Fractions in Adult and Geriatric Prostate. Journal of Radioanalytical and Nuclear Chemistry, 301, 383-397. https://doi.org/10.1007/s10967-014-3173-3

[23] Zaichick, V. (2015) The Variation with Age of 67 Macro- and Microelement Contents in Nonhyperplastic Prostate Glands of Adult and Elderly Males Investigated by Nuclear Analytical and Related Methods. Biological Trace Element Research, 168, 44-60. https://doi.org/10.1007/s12011-015-0342-3

[24] Zaichick, V. and Zaichick, S. (2015) Dietary Intake of Minerals and Prostate Cancer: Insights into Problem Based on the Chemical Element Contents in the Prostate Gland. Journal of Aging Research and Clinical Practice, 4, 164-171.

[25] Zaichick, V. and Zaichick, S. (2015) Global Contamination from Uranium: Insights into Problem Based on the Uranium Content in the Human Prostate Gland. Journal of Environmental Health Science, 1, 1-5.

[26] Zaichick, V. and Zaichick, S. (2016) Variations in Concentration and Distribution of Several Androgen-Dependent and -Independent Trace Elements in Nonhyperplastic Prostate Gland Tissue Throughout Adulthood. Journal of Andrology and Gynaecology, 4, 1-10.

[27] Zaichick, V. and Zaichick, S. (2016) Age-Related Changes in Concentration and Histological Distribution of $\mathrm{Br}, \mathrm{Ca}, \mathrm{Cl}, \mathrm{K}, \mathrm{Mg}, \mathrm{Mn}$, and $\mathrm{Na}$ in Nonhyperplastic Prostate of Adults. European Journal of Biology and Medical Science Research, 4, 31-48.

[28] Zaichick, V. and Zaichick, S. (2016) Variations in Concentration and Histological Distribution of $\mathrm{Ag}$, Co, Cr, Fe, Hg, Rb, Sb, Sc, Se, and $\mathrm{Zn}$ in Nonhyperplastic Prostate Gland Throughout Adulthood. Jacobs Journal of Cell and Molecular Biology, 2, 011.

[29] Zaichick, V. and Zaichick, S. (2016) Age-Related Changes in Concentration and Histological Distribution of 54 Trace Elements in Nonhyperplastic Prostate of Adults. International Archives of Urology and Complications, 2,019. https://doi.org/10.23937/2469-5742/1510019

[30] Zaichick, V. (2006) Medical Elementology as a New Scientific Discipline. Journal of Radioanalytical and Nuclear Chemistry, 269, 303-309. https://doi.org/10.1007/s10967-006-0383-3

[31] Stitch, S.R. (1957) Trace Elements in Human Tissue. I: A Semi-Quantitative Spectrographic Survey. Biochemistry Journal, 67, 97-103. https://doi.org/10.1042/bj0670097

[32] Tipton, J.H. and Cook, M.J. (1963) Trace Elements in Human Tissue. Part II. Adult 
Subjects from the United States. Health Physics, 9, 103-145. https://doi.org/10.1097/00004032-196302000-00002

[33] Györkey, F., Min, K.-W., Huff, J.A. and Györkey, P. (1967) Zinc and Magnesium in Human Prostate Gland: Normal, Hyperplastic, and Neoplastic. Cancer Research, 27, 1349-1353.

[34] Sangen, H. (1967) The Influence of the Trace Metals upon the Aconitase Activity in Human Prostate Glands. Japanese Journal of Urology, 58, 1146-1159.

[35] Schneider, H.-J., Anke, M. and Holm, W. (1970) The Inorganic Components of Testicle, Epididymis, Seminal Vesicle, Prostate and Ejaculate of Young Men. International Urology and Nephrology, 2, 419-427. https://doi.org/10.1007/BF02081698

[36] Hienzsch, E., Schneider, H.-J. and Anke, M. (1970) Vergleichende Untersuchungen zum Mengen- und Spurenelementgehalt der normalen Prostata, des Prostataadenoms und des Prostatakarzinoms. Zeitschrift für Urologie und Nephrologie, 63, 543-546.

[37] Soman, S.D., Joseph, K.T., Raut, S.J., Mulay, G.D., Parameswaran, M. and Pandey, V.K. (1970) Studies of Major and Trace Element Content in Human Tissues. Health Physics, 19, 641-656. https://doi.org/10.1097/00004032-197011000-00006

[38] Forssen, A. (1972) Inorganic Elements in the Human Body. I. Occurrence of Ba, Br, $\mathrm{Ca}, \mathrm{Cd}, \mathrm{Cs}, \mathrm{Cu}, \mathrm{K}, \mathrm{Mn}, \mathrm{Ni}, \mathrm{Sn}, \mathrm{Sr}, \mathrm{Y}$ and $\mathrm{Zn}$ in the Human Body. Annales Medicinae Experimentalis et Biologie, 50, 99-162.

[39] Dhar, N.K., Goel, T.C., Dube, P.C., Chowdhury, A.R. and Kar, A.B. (1973) Distribution and Concentration of Zinc in the Subcellular Fractions of Benign Hyperplastic and Malignant Neoplastic Human Prostate. Experimental and Molecular Pathology, 19, 139-142. https://doi.org/10.1016/0014-4800(73)90073-7

[40] Jafa, A., Mahendra, N.M., Chowdhury, A.R. and Kamboj, V.P. (1980) Trace Elements in Prostatic Tissue and Plasma in Prostatic Diseases of Man. Indian Journal of Cancer, 17, 34-37.

[41] Marezynska, A., Kulpa, J. and Lenko, J. (1983) The Concentration of Zinc in Relation to Fundamental Elements in the Diseases Human Prostate. International Urology and Nephrology, 15, 257-265. https://doi.org/10.1007/BF02083012

[42] Hienzsch, E., Schneider, H.-J., Anke, M., Hennig, A. and Groppel, B. (1979) The Cadmium-, Zinc-, Copper-, and Managanese-Level of Different Organs of Human Beings without Considerable Cd-Exposure Independence on Age and Sex. In: Anke, M. and Schneider, H.-J., Eds., Kadmiumsymposium, Wissenschaftliche Beiträge der Friedrich-Schiller-Universität, Jena, 276-282.

[43] Picurelli, L., Olcina, P.V., Roig, M.D. and Ferrer, J. (1991) Determination of Fe, Mg, $\mathrm{Cu}$, and $\mathrm{Zn}$ in Normal and Pathological Prostatic Tissue. Actas Urológicas Españolas, 15, 344-350.

[44] Galván-Bobadilla, A.I., García-Escamilla, R.M., Gutiérrez-García, N., MendozaMagaña, M.L. and Rosiles-Martínez, R. (2005) Cadmium and Zinc Concentrations in Prostate Cancer and Benign Prostate Hyperplasia. Revista Latinoamericana de Patología Clínica, 52, 109-117.

[45] Yaman, M., Atici, D., Bakirdere, S. and Akdeniz, I. (2005) Comparison of Trace Metal Concentrations in Malignant and Benign Human Prostate. Journal of Medicinal Chemistry, 48, 630-634. https://doi.org/10.1021/jm0494568

[46] Kwiatek, W.M., Banas, A., Banas, K., Podgorczyk, M., Dyduch, G., Falkenberg, G., Gajda, M. and Cichocki T. (2006) Distinguishing Prostate Cancer from Hyperplasia. Acta Physica Polonica, 109, 377-381. https://doi.org/10.12693/APhysPolA.109.377

[47] Guntupalli, J.N.R., Sarita, P., Ramana Murty, G.A.V., Ravi Kumar, M., Seetharami 
Reddy, B., Lakshminarayana, S., Prema Chand, K., Durga Prasad, A., Bhuloka Reddy, S., Vijayan, V., Rama Lakshmi, P.V.B. and Satyanarayana, G. (2007) Trace Elemental Analysis of Normal, Benign Hypertrophic and Cancerous Tissues of the Prostate Gland Using the Particle-Induced X-Ray Emission Technique. European Journal of Cancer Prevention, 16, 108-115. https://doi.org/10.1097/01.cej.0000228409.75976.b6

[48] Tohno, S., Kobayashi, M., Shimizu, H., Tohno, Y., Suwannahoy, P., Azuma, C., Minami, T., Sinthubua, A. and Mahakkanukrauh P. (2009) Age-Related Changes of the Concentrations of Select Elements in the Prostates of Japanese. Biological Trace Element Research, 127, 211-227. https://doi.org/10.1007/s12011-008-8241-5

[49] Kiziler, A.R., Aydemir, B., Guzel, S., Alici, B., Ataus, S., Tuna, M.B., Durak, H. And Kilic, M. (2010) May the Level and Ratio Changes of Trace Elements Be Utilized in Identification of Disease Progression and Grade in Prostatic Cancer? Trace Elements and Electrolytes, 27, 65-72. https://doi.org/10.5414/TEP27065

[50] Zaichick, S. and Zaichick, V. (2010) Method and Portable Facility for Energy-Dispersive X-Ray Fluorescent Analysis of Zinc Content in Needle-Biopsy Specimens of Prostate. X-Ray Spectrometry, 39, 83-89. https://doi.org/10.1002/xrs.1233

[51] Zaichick, V. and Zaichick, S. (2013) The Effect of Age on Br, Ca, Cl, K, Mg, Mn, and $\mathrm{Na}$ Mass Fraction in Pediatric and Young Adult Prostate Glands Investigated by Neutron Activation Analysis. Applied Radiation and Isotopes, 82, 145-151. https://doi.org/10.1016/j.apradiso.2013.07.035

[52] Zaichick, V. and Zaichick, S. (2013) NAA-SLR and ICP-AES Application in the Assessment of Mass Fraction of 19 Chemical Elements in Pediatric and Young Adult Prostate Glands. Biological Trace Element Research, 156, 357-366. https://doi.org/10.1007/s12011-013-9826-1

[53] Leitão, R.G., Palumbo, A., Souza, P.A.V.R., Pereira, G.R., Canellas, C.G.L., Anjos, M.J. and Nasciutti, L.E. (2014) Elemental Concentration Analysis in Prostate Tissues Using Total Reflection X-Ray Fluorescence. Radiation Physics and Chemistry, 95, 62-64. https://doi.org/10.1016/j.radphyschem.2012.12.044

[54] Zaichick, S. and Zaichick, V. (2014) EDXRF Determination of Trace Element Contents in Benign Prostatic Hypertrophic Tissue. In: Frank, I.M. and Shapiro, F.L., Eds., Fundamental Interactions and Neutrons, Neutron Spectroscopy, Nuclear Structure, Ultracold Neutrons, Related Topics, Joint Institute for Nuclear Research, Dubna, 311-316.

[55] Denoyer, D., Clatworthy, S.A.S., Masaldan, S., Meggyesy, P.M. and Cater, M.A. (2015) Heterogeneous Copper Concentrations in Cancerous Human Prostate Tissues. Prostate, 75, 1510-1517. https://doi.org/10.1002/pros.23022

[56] Zaichick, S. and Zaichick, V. (2015) Prostatic Tissue Level of Some Androgen Dependent and Independent Trace Elements in Patients with Benign Prostatic Hyperplasia. Andrology and Gynecology: Current Research, 3, 3. https://doi.org/10.4172/2327-4360.1000141

[57] Singh, B.P., Dwivedi, S., Dhakad, U., Murthy, R.C., Choubey, V.K., Goel, A. and Sankhwar, S.N. (2016) Status and Interrelationship of Zinc, Copper, Iron, Calcium and Selenium in Prostate Cancer. Indian Journal of Clinical Biochemistry, 31, 50-56. https://doi.org/10.1007/s12291-015-0497-x

[58] Zaichick, V. and Zaichick, S. (2016) Trace Element Contents in Adenocarcinoma of Human Prostate Investigated by Energy Dispersive X-Ray Fluorescent Analysis. Journal of Adenocarcinoma, 1, 1-7.

[59] Zaichick, V. and Zaichick, S. (2016) The Bromine, Calcium, Potassium, Magnesium, Manganese, and Sodium Contents in Adenocarcinoma of Human Prostate Gland. 
Journal of Hematology and Oncology Research, 2, 1-12. https://doi.org/10.14302/issn.2372-6601.jhor-15-896

[60] Zaichick, V. and Zaichick, S. (2016) Trace Element Contents in Adenocarcinoma of the Human Prostate Gland Investigated by Neutron Activation Analysis. Cancer Research and Oncology, 1, 1-10.

[61] Zaichick, V. and Zaichick, S. (2016) Prostatic Tissue Levels of 43 Trace Elements in Patients with Prostate Adenocarcinoma. Cancer and Clinical Oncology, 5, 79-94. https://doi.org/10.5539/cco.v5n1p79

[62] Zaichick, V. and Zaichick, S. (2016) Prostatic Tissue Level of Some Major and Trace Elements in Patients with BPH. Jacobs Journal of Nephrology and Urology, 3, 026.

[63] Zaichick, V. and Zaichick, S. (2016) Levels of 43 Trace Elements in Hyperplastic Prostate Tissues. British Journal of Medicine and Medical Research, 15, 1-12. https://doi.org/10.9734/BJMMR/2016/25275

[64] Zaichick, V. and Zaichick, S. (2016) Chemical Elemental Content/Calcium Ratios in Tissues of Human Hyperplastic Prostate Gland. Journal of Applied Life Sciences International, 4, 1-11. https://doi.org/10.9734/JALSI/2016/24747

[65] Zaichick, V., Sviridova, T. and Zaichick, S. (1997) Zinc in Human Prostate Gland: Normal, Hyperplastic and Cancerous. International Urology and Nephrology, 29, 565-574. https://doi.org/10.1007/BF02552202

[66] Zaichick, S. and Zaichick, V. (2012) Trace Elements of Normal, Benign Hypertrophic and Cancerous Tissues of the Human Prostate Gland Investigated by Neutron Activation Analysis. Applied Radiation and Isotopes, 70, 81-87. https://doi.org/10.1016/j.apradiso.2011.08.021

[67] Zaichick, V. (1997) Sampling, Sample Storage and Preparation of Biomaterials for INAA in Clinical Medicine, Occupational and Environmental Health. In: IAEA, Ed., Harmonization of Health-Related Environmental Measurements Using Nuclear and Isotopic Techniques, IAEA, Vienna, 123-133.

[68] Zaichick, V. and Zaichick, S. (1996) Instrumental Effect on the Contamination of Biomedical Samples in the Course of Sampling. The Journal of Analytical Chemistry, 51, 1200-1205. (In Russian)

[69] Zaichick, V. and Zaichick, S. (1997) A Search for Losses of Chemical Elements during Freeze-Drying of Biological Materials. Journal of Radioanalytical and Nuclear Chemistry, 218, 249-253. https://doi.org/10.1007/BF02039345

[70] Zaichick, V. (2004) Losses of Chemical Elements in Biological Samples under the Dry Aching Process. Trace Elements in Medicine, 5, 17-22. (In Russian)

[71] Korelo, A.M. and Zaichick, V. (1993) Software to Optimize the Multielement INAA of Medical and Environmental Samples. In: Nazarov, V.M., Ed., Activation Analysis in Environment Protection, Joint Institute for Nuclear Research, Dubna, 326-332. (In Russian)

[72] Catalona, W.J. (1996) Clinical Utility of Measurements of Free and Total Prostate-Specific Antigen (PSA): A Review. Prostate, 29, 64-69. https://doi.org/10.1002/(SICI)1097-0045(1996)7+<64::AID-PROS9>3.0.CO;2-J

[73] Genes V.S. (1967) Simple Methods for Cybernetic Data Treatment of Diagnostic and Physiological Studies. Nauka, Moscow, 208 p. (In Russian) 
Submit or recommend next manuscript to SCIRP and we will provide best service for you:

Accepting pre-submission inquiries through Email, Facebook, LinkedIn, Twitter, etc. A wide selection of journals (inclusive of 9 subjects, more than 200 journals)

Providing 24-hour high-quality service

User-friendly online submission system

Fair and swift peer-review system

Efficient typesetting and proofreading procedure

Display of the result of downloads and visits, as well as the number of cited articles Maximum dissemination of your research work

Submit your manuscript at: http://papersubmission.scirp.org/

Or contact jet@scirp.org 